\title{
Use of oxygen-ozone therapy in the treatment of fibromyalgia
}

\author{
Rossella Balestrero, ${ }^{1,2}$ Marianno Franzini, ${ }^{2}$ Luigi Valdenassi ${ }^{2,3}$ \\ ${ }^{1}$ Medical Surgeon; ${ }^{2}$ Oxygen-Ozone Therapy Scientific Society, Gorle (BG); ${ }^{3}$ Toxicology School, University of Pavia, \\ Italy
}

\begin{abstract}
Fibromyalgia (FM) is a pathology that is manifested by a spectrum of heterogeneous symptoms that are difficult to frame, from both a diagnostic and therapeutic point of view. On one hand, the clinical features are highly variable, and on the other, the pathophysiology has yet to be fully clarified. This paper aims to highlight how oxygen ozone - administered using a precise protocol from the Scientific Society of Oxygen Ozone Therapy - is able to act on several levels of the pathophysiology of FM by virtue of its intrinsic biochemical mechanisms, making it a viable option for treating the disease. The following case study a 45 year old woman who came to our attention due to diffuse pain and a feeling of general tiredness - responded positively to a standard treatment with oxygen-ozone therapy delivered via bi-weekly sessions, for a total of 12 sessions from September to October 2016. The treatment was followed by a maintenance therapy of about one session a month. One month after the end of the 12 sessions, the patient reported a significant improvement in pain, mood, and a significant reduction in fatigue, a new feeling of well-being and an improved quality of sleep. In the light of these results, oxygen-ozone therapy emerges as a viable therapeutic option for the treatment of FM patients.
\end{abstract}

\section{Introduction}

\section{Fibromyalgia}

Fibromyalgia (FM) is a complex disease that is manifest by a spectrum of heterogeneous symptoms that are difficult to frame,

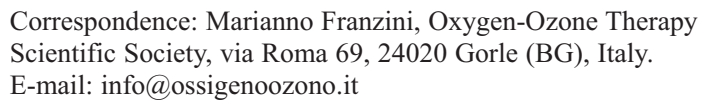

This article is distributed under the terms of the Creative Commons Attribution Noncommercial License (by-nc 4.0) which permits any noncommercial use, distribution, and reproduction in any medium, provided the original author(s) and source are credited. from both a diagnostic point of view and as regards which therapeutic approach to use. This is due to the fact that the clinical features are highly variable, and the pathophysiology has not yet been fully clarified.

With regard to the symptomatology, FM presents itself in most patients by three key symptoms that can be present individually or in various combinations. These are: chronic widespread pain and pain elicited by pressure on trigger points, fatigue, and sleep disorders (in particular trouble getting to sleep or non-restorative sleep). In addition to these symptoms, which occur with an intensity that varies from patient to patient, but can exhibit fluctuations over time, even in the same subject, we also find mood disorders, stiffness, difficulty concentrating or memory disorders, migraines and other somatic complaints. ${ }^{1,2}$ Given this wide variability in the clinical presentation of FM, it is now considered as more of a complex range of problems, rather than a single disease.

In addition to the heterogeneity in clinical manifestations, diagnosis is rendered difficult by the fact the pathophysiology of FM is not completely understood, and as a result, the therapies currently available do not appear completely effective in most cases. Various studies have shown that numerous mechanisms take part in the genesis of this disease, including alterations in the processing of painful stimulus at a central level, neurotransmitter alterations and hypothalamic-pituitary axis changes, as well as an increased level of several cytokines. ${ }^{3}$ Finally, in recent years, oxidative stress has assumed a leading role in the pathophysiology of $\mathrm{FM}^{4}$ although it is not yet known whether its increase is a causative factor in the disease or a consequence of the patient's general condition. ${ }^{5,6}$ In patients with FM, an increase in the concentrations of malonyldialdehyde have been found, alongside a low concentration of antioxidant enzymes like catalase, glutathione peroxidase and superoxide dismutase..$^{5,7}$

Ozone is known to exert a mild, transient and controlled oxidative stress that promotes upregulation of antioxidant systems and modulation of the immune system. ${ }^{8}$

In accordance with this its mechanism of action is suggested as an effective therapeutic indication in the treatment of FM.

\section{Purpose}

The aim of this paper is to highlight how ozone-oxygen delivered using a precise SIOOT protocol, thanks to its intrinsic biochemical mechanisms, can act on several levels of FM pathophysiology, rendering it a viable option for treating the disease.

\section{Fibromyalgia Survey Questionnaire and Fibromyalgia Impact Questionnaire-Revised}

Because the diagnosis and FM evaluation are predominantly clinical, the results, in terms of treatment efficacy, were evaluated by comparing the FSQ (Fibromyalgia Survey Quesionnaire) and FIQR (Fibromyalgia Impact Questionnaire-Revised) question- 
naires compiled by the patient before and after the ozone therapy cycle. The FSQ is a questionnaire that uses the diagnostic criteria set by the American College of Rheumatology ${ }^{9}$ and it is composed of two different rating scales, to each of which is assigned a score, which when exceeded, indicates a diagnosis of FM. The two scales were the Widsperead Pain Index and Simptoms Severity Scale. The first serves to highlight the presence of pain in 19 parts of the body. The possible scores on this scale are 0 to 19. The second scale takes into account the other symptoms of FM, like sleep disturbance and fatigue as well as the somatic symptoms most commonly found in these patients. Scores range from 0 to 12 . The final diagnosis is made when WPI $>7$ out of 19 sites and $\mathrm{SSS}>5$, or WPI between 4 and 6 and $\mathrm{SSS}>9$, generalised pain must also be present, and the symptoms must have persisted for $>3$ months. ${ }^{9,10}$ Next to this, to evaluate how effective oxygen-ozone therapy is at reducing pain symptoms, we asked the patient to record, for each region selected using the WPI, the level of pain intensity perceived by her, before and after therapy, using a visual analogic pain scale (VAS).

The FSQ questionnaire, as well as being a valuable tool for diagnosing FM, may also be useful for assessing the severity of the clinical picture. Both scores, on the SSS and WPI scales in fact, correlate directly with the patient's general status, and both scores can be added together to give a score from 0-31 which gives an idea of the severity of the clinical condition. ${ }^{10}$ However, to complete the assessment of the patient in this study, we also used the FIQR questionnaire, a tool used to assess the degree of general disability from which deductions can be made regarding the affected individual's quality of life. ${ }^{11}$ On the basis of this questionnaire, the score was divided into mild 0-39, moderate 39-59 and severe 59100. A device was used for delivery [model: Medical Ozone 95 CPS from Multiossigen Srl [Gorle (BG), Italy] and for Autologous hemotherapy a kit (model: SANO3), specifically certified for use with blood and ozone.

\section{Case Report}

\section{Description}

The propositus was a 45-year-old female, who came to our attention because of diffuse pain and a feeling of general fatigue that severely limited her daily activities, to the extent that it became necessary to take a leave of absence from work. The symptoms were reported to have onset at about 20 years of age, with an exacerbation after the age of 30 .

The past and recent medical history is negative for other diseases that could potentially be the cause of these symptoms. Blood tests show only a slight increase in the levels of anti-EBV antibodies.

The patient reported that they had been subjected to numerous other tests by different specialists without receiving a precise definition of their disorder. Treatments offered had included the use of muscle relaxants and gabapentinoids, but had never had any effect, and had to be abandoned after a short time. At the beginning of 2016, a diagnosis of FM was suggested, and in September of the same year the patient was seen by a consultant in oxygen-ozone therapy.

At our centre, a full examination was performed and the patient was asked to fill out the FSQ questionnaire which confirmed a diagnosis of FM: Score SSS=7, WPI=15 (total score=22). The patient then filled out the FIQR questionnaire with a final score of 58 , indicating a moderate severity. From the case history, the visits and the questionnaires, the following profile emerged.

The patient was evidently suffering, with ever-present widespread pain in the neck and upper back. Stiffness, with functional limitations in executing large movements, and easy fatigability.
They reported high levels of fatigue, and limitations in performing normal daily activities like working or doing housework for a long period, particularly because of the pain. They reported non-restorative sleep, and difficulty falling asleep. The patient clearly displayed the characteristics of an anxiety-depressive trait, which she confirmed herself in the questionnaire. The anxiety component definitely appeared to be dominant. Reduced, albeit slightly, ability to concentrate. The patient also complained of numbness in the hands and feet. A week before the meeting, i.m. Voltaren ${ }^{\circledR}$ injections were administered because of an exacerbation of painful symptoms, and then suspended as not effective.

From the interview a major sense of frustration emerged, mainly due to the fact there had been no improvement with any of the therapies performed up to that point. The partial benefit obtained from physical activity has failed at a time when, due to the severe pain, the patient could no longer perform it. The patient is then given an explanation about the rationale for using ozone therapy and, in the absence of contraindications; a treatment plan is suggested that the patient agrees to carry out.

\section{Oxygen-ozone therapy}

From September 2016 to October 2016, the patients underwent a first cycle of 12 biweekly sessions of GAE. These used $150 \mathrm{cc}$ of a mixture per $150 \mathrm{~mL}$ of blood, at increasing concentrations from 30 $\mathrm{mg} / \mathrm{mL}$ up to $50 \mu \mathrm{g} / \mathrm{mL}$ after the first two sessions, alternate with PAE $10 \mathrm{cc}$. blood and $10 \mathrm{cc}$ oxygen-ozone and rectal insufflation. Proceeding then with a monthly maintenance. At the end of treatment, the patient was asked to fill out the FSQ (always with the VAS scale associated with WPI) and FIQR questionnaires again.

\section{Results}

At the end of the therapeutic cycle, the patient continued with a monthly maintenance. One month after the end of 12 sessions, a marked improvement in pain and stiffness was reported, which remained to a very limited extent in those areas previously reported as the most painful, and almost disappeared in others. We noted next to this, a significant reduction in fatigue, a general feeling of well-being and an improved quality of sleep. The mood was also clearly improved, despite the anxiety still being evident. Paresthesias are also omitted. In addition to a clearly lower FSQ questionnaire score, with a score $\mathrm{WPI}=7$ and $\mathrm{SSS}=1$ (total score $=8$ ), we also saw a reduction in values obtained using the VAS scale in individual areas of the body that evidenced a reduction of pain very well, and a decrease in the FIQR questionnaire score which fell to a value of 46 . These factors indicated to us as a whole, that we had achieved a marked improvement in the severity of the clinical picture and consequently in the patient's quality of life in general.

\section{Discussion}

\section{Pathophysiology of fibromyalgia}

FM is a chronic disorder with a very complex pathophysiology. ${ }^{5}$ Despite generalised pain being considered the cardinal symptom, others like tiredness and being easily fatigued, non-restorative sleep and mood disorders in the sense of anxietydepression, play a significant role in determining the degree of disability characteristic of this disease. ${ }^{5}$ Even the pathogenesis of FM is very complex and, as mentioned earlier, many factors converge 
to determine it. These include among others, alterations in the pain transmission-, neuro-hormonal and immune systems. ${ }^{3}$ Even the increase in cytokines appears to have a role in the genesis of FM symptoms. ${ }^{12}$ In affected patients in particular, an increased level of certain cytokines (including IL-6 and IL-8, the release of which in turn is probably stimulated by other systems upstream like the sympathetic nervous system, could intervene in causing the symptoms of FM. In fact, as is known, IL-8 promotes sympathetic pain and IL-6 induces hyperalgesia, fatigue and depression. ${ }^{12}$

More recently, there has been some evidence for the dysregulation of oxidative processes playing a role, in studies that have shown the plasma of FM patients contains increased levels of lipid peroxidation products (LP) and carboxylated proteins, the end result of both being membrane damage induced by ROS, 4,6,13 alongside a reduction in total antioxidant capacity or of antioxidant enzymes such as superoxide dismutase (SOD) and catalase. ${ }^{4,6,13}$ Several experimental models have revealed an increase in the production - on several levels - of ROS and other harmful radicals, which go on to interact with cellular structures, resulting in the typical symptoms of FM. Oxidative stresses and ROS such as for example, NO seem therefore to be mainly involved in the pathogenesis of FM even if it is not yet known whether redox equilibrium alterations are a cause or effect of this pathology.5,6

Studies to date have shown how various mechanisms come into play in the body. For example, the cause of $\mathrm{H} 2 \mathrm{O} 2$ increases in the neutrophils of these patients, similar to an increase of superoxide of mitochondrial origin in the mononuclear cells of the peripheral blood, ${ }^{4,14}$ can be explained by a CoQ10 deficiency in patients with $\mathrm{FM}^{4}$ CoQ10 is a vital element in the respiratory chain, and its reduction leads to a decrease in the expression of proteins involved in mitochondrial oxidative phosphorylation, and to a reduction in mitochondrial membrane potential and an increase in ROS production. ${ }^{14}$

From a physiological point of view also, we know that the production of LP reflects precisely the intracellular ROS increase which in turn is involved in the etiology of one of the major symptoms of FM: pain. ${ }^{4}$ In fact, for example, superoxide radicals play a role in the genesis of pain, both by sensitizing the peripheral and central nervous systems, and thereby altering nociception, as well as by activating the release of certain cytokines, such as IL- $8 .{ }^{4} \mathrm{NO}$ also promotes the excessive release of excitatory amino acids and substance P from presynaptic terminals afferent to the dorsal horn of the spinal cord, which are hyperstimulated, ${ }^{5,15}$ which seems to partly explain the alterations in processing the painful stimulus. ${ }^{5,16}$

At the same time, there were also effects at the level of the muscle layer. In fact, damage to the sarcolemma produced by ROS would cause pain as well as muscle stiffness, ${ }^{7}$ so much that there were significant correlations between the levels of antioxidants (in both serum and plasma) and the pains and stiffness, as assessed by appropriate measurement scales. ${ }^{4,13,14}$ Furthermore, oxidative stress induces an alteration of muscle metabolism with increases in intracellular calcium and consequently the loss of potassium from the sarcomere: therefore sensitisation of the nociceptors increases, and this results once more in pain, weakness and muscle stiffness. ${ }^{5,17}$ In these patients as a rule, therefore, structural alterations are observed in muscle, a factor which further reduces tissue oxygenation, leading to the accumulation of agents that peroxidise the lipid membrane, ${ }^{5}$ thus establishing a self-fuelling vicious circle.

Accumulation of LP on the other hand, has been linked to another typical symptom of FM: fatigue. ${ }^{4}$ High levels of LP have been demonstrated in chronic fatigue syndrome, a condition that has a high level of comorbidity with FM. ${ }^{4}$ It also seems likely that it plays a role in the induction of depression. ${ }^{18}$ All these data confirm a role for oxidative stress in the genesis of FM.

\section{Mechanisms of action of ozone}

Oxygen-ozone therapy is a method that, by using a gas mixture called medical oxygen, exploits the inherent properties of ozone same to invoke a moderate and transient oxidative stress when the ozone comes into contact with various parts of the body's biochemistry. ${ }^{19-22}$ Nowadays, the paradoxical action of ozone - which despite being an oxidizing molecule, actually increases the antioxidant properties of the main redox systems - are well known. ${ }^{19-21}$ The mechanism behind ozone's paradoxical action is as follows.

Soon after coming into contact with the blood, the ozone dissolves and reacts immediately with various reducing molecules like unsaturated fatty acids containing double bond (PUFA's), antioxidizing agents and other molecules, to generate reactive oxygen species (ROS). ${ }^{19,23-26}$ In particular, its reaction with PUFA's generates LP and hydrogen peroxide. The toxicity of these molecules is largely neutralised by enzymatic antioxidant systems like glutathione-transferase and aldehyde dehydrogenase, which are commonly present in our body, while the products of these reactions act as secondary messengers that stimulate further synthesis of antioxidant enzymes. ${ }^{19,22,24}$ Obviously, the ozone must be administered in quantities that allow us to achieve a therapeutic effect that protects against radicals, and is non-toxic; the concentrations commonly used for medical purposes are fully compatible with this. Ozone also has effects on lipid, carbohydrate and protein metabolism, and induces a reduction in circulating cytokines and therefore of the inflammatory state. Besides that, ozone can also improve the circulation and therefore tissue oxygenation, helping to reduce ischaemic/hypoxic states. ${ }^{27}$

\section{Therapeutic suggestions}

Current guidelines suggest a combination of drug- and nondrug therapy, where the latter term primarily means patient education, exercise and cognitive behavioural therapy. ${ }^{28}$ As for the drugs chosen for treating this disease, those for which there is most evidence are pregabalin, duloxetine and milnacipran, followed by amitriptyline and cyclobenzaprine. ${ }^{29}$ Other drugs with at least one positive clinical trial are for example, several selective serotinin uptake inhibitors, gabapentin and tramadol. ${ }^{29}$ However, none of the currently available drugs are fully effective across the entire spectrum of FM symptoms, which include pain, fatigue, sleep disturbances and depression. ${ }^{29}$ Therapeutic combinations of these drugs are another option that has yet to be further investigated in clinical trials. ${ }^{29}$

In addition, with regard to non-drug options the entity responding to these therapies often exceeds results obtained with the drugs, ${ }^{28}$ which, apart from being ineffective, are also burdened by side effects. The greatest benefit was seen in an overall functional improvement, and these results were confirmed over time. ${ }^{28,30,31}$ However, availability, compliance, and maintenance over the long term are the main limitations for these forms of therapy. ${ }^{28}$ EULAR, ${ }^{32}$ the latest review of FM treatment, emphasises the need to properly inform and educate patients about their disease and the usefulness of moderate aerobic exercise, such as yoga or meditation, amongst others. There are, however, studies that have compared various physical activities in an effective way to determine which is best when the choice is based on the patient's own abilities.

In accord with these mechanisms of action, oxygen-ozone therapy is a valid therapeutic option for treating FM, and is suitable in a panorama where both the poor efficacy of the treatments proposed up to now, plus the extreme variability of the clinical picture requires a continuous adjustment of therapy, making it difficult to maintain high patient compliance. Some studies have investigated the effectiveness of ozone for treating FM, both via GAE27 or via rectal insufflation..$^{5}$ These have reported promising 
results with high percentage response rates in treated patients. This is an improvement from the clinical point of view, which involved not only the pain aspect of the condition, but also a general improvement of other conditions, including mood. Improvements were also evident from a compliance perspective: the treatment had no side effects and, with a maintenance time of approximately one session per month, it was very well tolerated by almost all of the patients.

\section{Conclusions}

The case we followed responded positively to a standard treatment with oxygen-ozone therapy administered via biweekly GAE sessions, for a total of 12 sessions, followed by a maintenance treatment of about one session per month.

In view of the findings of studies on the pathophysiology of FM, as well as the biochemical properties of ozone, ozone-oxygen therapy thus appears to definitely be a valid therapeutic option for the treatment of patients with FM. Due to the poor efficacy of the drugs used to date, in fact and aggravated by the presence of numerous side effects, there does not appear to be an adequate treatment for the disease. ${ }^{33-35}$ In addition, studies conducted so far with non-drug therapies, such as exercise and cognitive-behavioural therapy, even though some have reported positive results, do not provide accurate guidelines or indicate the most effective practice from the others.

All these factors greatly reduce the patient's adherence to treatment, and those who found no improvement in their quality of life, have tended to abandon them. This further fuels their depressive state. With oxygen-ozone therapy however, the patient experiences a feeling of general well-being, due certainly to a reduction of the painful symptoms, which was also well demonstrated in our clinical case, but is also the result of an improvement in the asthenia. This is due to a greater oxygenation of tissues caused by the ozone. The improvements in mood and quality of sleep are also important factors in the overall status of these patients.

Apart from this, there were no side effects in this case or in any of the patients treated in larger trials ${ }^{5,27}$ and the degree of compliance with therapy was total.

The main limitation of studies carried out in this area to date is the small number of patients on which they were conducted. The hope is that, thanks to the greater spread of this medical practice, it will be possible to undertake more studies to encourage use in a wider number of patients.

In this paper, we have taken the opportunity of presenting a clinical case we treated successfully to compile a review about FM and its treatment with oxygen-ozone therapy, to confirm, once again, its effective therapeutic efficacy.

\section{References}

1. Mease P, Arnold LM, Choy EH, et al. Fibromyalgia syndrome module at OMERACT 9: domain construct. J Rheumatol 2009;36:2318-29.

2. Mease P. Fibromyalgia syndrome: review of clinical presentation, pathogenesis, outcome measures, and treatment. J Rheumatol 2005;32:6-21.

3. Howard S, Smith MD, Harris R, Clauw D. Fibromyalgia: an afferent processing disorder leading to a complex pain generalized syndrome. Pain Physician 2011;14:217-45.

4. Cordero MD. Oxidative stress in fibromyalgia: pathophysiolo- gy and clinical implications. Reumatol Clin 2011;7:281-3.

5. Hidalgo-Tallon J, Menendez-Cepero S, Vilchez JS, et al. Ozone therapy as add-on treatment in fibromyalgia management by rectal insufflation: an open-label pilot study. J Altern Complement Med 2013;19:238-42.

6. Ozgocmen S, Ozyurt H, Sogut S, et al. Antioxidant status, lipid peroxidation and nitric oxide in fibromyalgia: etiologic and therapeutic concerns. Rheumatol Int 2006;26:598-603.

7. Iqbal R, Mughal MS, Arshad N, et al. Pathophysiology and antioxidant status of patients with fibromyalgia. Rheumatol Int 2011;31:149-52.

8. Bocci V, Di Paolo N. Oxygen-ozone therapy in medicine: an update. Blood Purif 2009;28:373-6.

9. Wolfe F, Clauw DJ, Fitzcharles MA, et al. The American College of Rheumatology preliminary diagnostic criteria for fibromyalgia and measurement of symptom severity. Arthr Care Res 2010;62:600-10.

10. Häuser W, Ablin J, Perrot S, Fitzcharles MA. Management of fibromyalgia: key messages from recent evidence-based guidelines. Polish Arch Int Med 2017;2017:127.

11. Bennett RM, Friend R, Jones KD, et al. The revised fibromyalgia impact questionnaire (FIQR): validation and psychometric properties. Arthritis Res Ther 2009;11:R120.

12. Wallace DJ, Linker-Israeli M, Hallegua D, et al. Weisman cytokines play an aetiopathogenetic role in fibromyalgia: a hypotesis and pilot study. Reumathology 2001;40:743-9.

13. Altindag O, Celik H. Total antioxidant capacity and the severity of the pain in patients with fibromyalgia. Redox Rep 2006;11:131-5.

14. Cordero MD, De Miguel M, Moreno Fernández AM, et al. Mitochondrial dysfunction and mitophagy activa- tion in blood mononuclear cells of fibromyalgia patients: implications in the pathogenesis of the disease. Arthritis Res Ther 2010;12:R17.

15. Schaible HG, Ebersberger A, von Banchet GS. Mechanisms of pain in arthritis. Ann NY Acad Sci 2001;966:343-54.

16. Staud R, Vierck CJ Jr, Cannon RC, et al. Abnormal sensitization and temporal summation of second pain (wind-up) in patients with fibromyalgia syndrome. Pain 2001;91:165-75.

17. Bengtsson A. The muscle in fibromyalgia. Rheumatology 2002;41:721-4.

18. Al-Gebouri AG, Al-Mashhadani Z, Al-Osami MH, Turki KM. Lipid peroxidation and oxidative stress in patients with fibromyalgia syndrome. Iraqi Postgr Med J 2011;10:101-5.

19. Valdenassi L, Franzini M, Simonetti V, Ricevuti G. Oxygenozone therapy: paradoxical stimulation of ozone. Ozone Therapy 2016;1:5837.

20. Muñoz A. Design and analysis of studies of the health effects of ozone. Environ Health Perspect 1993;101:231-5.

21. Plopper CG, Duan X, Buckpitt AR, Pinkerton KE. Dosedependent tolerance to ozone. Site-specific elevation in antioxidant enzymes in the lung of rats exposed for 90 days or 20 months. Toxicol Appl Pharmacol 1994;127:124-31.

22. Bocci V, Borrelli E, Travagli V, Zanardi I. The ozone paradox: ozone is a strong oxidant as well as a medical drug. Med Res Rev 2009;29:646-82.

23. Bocci V. Ozone therapy today. Proc. 12th World Congress of the International Ozone Association. Ozone in Medicine. Lille: Tours- Instaprint; 1995. pp 13-27.

24. Stone JR, Yang S. Hydrogen peroxide: a signaling messenger. Antioxid Redox Signal 2006;8:243-70.

25. Rilling SH. The basic clinic applications of ozone therapy. OzoNachrichten 1985;1/2:7-17.

26. Viebahn R. The use of ozone in Medicine. Berlin; Haugh Publ.: 1994. 
27. Borrelli E, Bocci V. A novel therapeutic option for chronic fatigue syndrome and fibromyalgia. Rivista Italiana di Ossigeno-Ozonoterapia 2002;1:149-53.

28. Daniel J, Clauw MD. Fibromyalgia: a clinical review. J Am Med Assoc 2014;311:15.

29. Williams DA, Cary MA, Groner KH, et al. Improving physical functional status in patients with fibromyalgia: a brief cognitive behavioral intervention. J Rheumatol 2002;29:1280-6.

30. Goldenberg DL, Burckhardt C, Crofford L. Management of fibromyalgia syndrome. J Am Med Assoc 2004;292:2388-95.

31. Macfarlane GJ, Kronisch C, Dean LE, et al. EULAR revised recommendations for the management of fibromyalgia. Ann
Rheum Dis 2016. Available from: ard.bmj.com/ content/early/2016/07/04/annrheumdis-2016-209724.full.pdf

32. Calandre EP, Rico-Villademoros F, Slim M. An update on pharmacotherapy for the treatment of fibromyalgia. Exp Opin Pharmacother 2015;16:1347-68.

33. Calandre EP, Rico-Villademoros F, Slim M. Pharmacological treatment of fibromyalgia: is the glass half empty or half full? Future Medicine. Pain Manage 2017;7:5-10.

34. Bennett RM. Pain management in fibromyalgia. Fut Med 2016;6:313-6.

35. Häuser WF. Wolfe diagnosis and diagnostic tests for fibromyalgia (syndrome). Reumatismo 2012;64:194-205. 